\section{Intersecting Paths of Emerging and Reemerging Infectious Diseases}

\author{
Tais M. Wilson, Christopher D. Paddock, \\ Sarah Reagan-Steiner, Julu Bhatnagar, Roosecelis \\ B. Martines, Andrea L. Wiens, Michael Madsen, \\ Kenneth K. Komatsu, Heather Venkat, Sherif R. Zaki
}

Author affiliations: Universidade de Brasília, Brasília, Brazil (T.M. Wilson); Centers for Disease Control and Prevention, Atlanta, Georgia, USA (T.M. Wilson, C.D. Paddock, S. ReaganSteiner, J. Bhatnagar, R.B. Martines, H. Venkat, S.R. Zaki); Pinal County Office of the Medical Examiner, Florence, Arizona, USA (A.L. Wiens); Coconino County Health and Human Services Medical Examiner's Office, Flagstaff, Arizona, USA (M. Madsen); Arizona Department of Health Services, Phoenix, Arizona, USA (K.K. Komatsu, H. Venkat)

DOI: https://doi.org/10.3201/eid2705.204779

Severe acute respiratory syndrome coronavirus 2 (SARSCoV-2) shares common clinicopathologic features with other severe pulmonary illnesses. Hantavirus pulmonary syndrome was diagnosed in 2 patients in Arizona, USA, suspected of dying from infection with SARS-CoV-2. Differential diagnoses and possible co-infections should be considered for cases of respiratory distress during the SARS-CoV-2 pandemic.

$\bigcirc$ evere acute respiratory syndrome coronavirus 2 (SARS-CoV-2), the virus that causes coronavirus disease (COVID-19), emerged in Wuhan, China, during December 2019 and spread rapidly to other parts of China and the world (1). However, the clinical and pathologic features of COVID-19 are also found for other respiratory disease, such as hantavirus pulmonary syndrome (HPS). In 1993, a hantavirus (Sin Nombre virus) and its rodent reservoir (Peromyscus maniculatus deer mouse) were identified as the causative agent and vertebrate reservoir responsible for an outbreak of severe pulmonary illness, named HPS, in the Four Corners region in the southwestern United States (2-4).

Soon after the emergence and recognition of COVID-19 in the United States in early 2020, the Infectious Diseases Pathology Branch, Division of High-Consequence Pathogens and Pathology, National Center for Emerging and Zoonotic Infectious Diseases, Centers for Disease Control and Prevention initiated diagnostic testing of fixed tissue specimens from deceased persons who had suspected or confirmed SARS-CoV-2 infection $(5,6)$. During
May 2020, Infectious Diseases Pathology Branch received tissues from an 11-year-old child (patient 1) from Arizona, who died after a brief illness culminating in severe respiratory distress. Histopathological findings included diffuse alveolar damage with rare hyaline membranes, intraalveolar edema, leukocytosis with a left shift (Figure, panel A), interstitial pneumonitis and immunoblasts in the red pulp and periarteriolar sheaths of the spleen (Figure, panel B). RNA extracted from formalin-fixed, paraffin-embedded trachea and lung tissues was positive for SARS-CoV-2 by conventional reverse transcription PCR (RT-PCR) and sequencing of positive amplicons. However, evaluation for SARSCoV-2 by using an immunohistochemical (IHC) assay (5) showed negative results.

Subsequently, embalmed lung tissues were received from the child's mother, a 25-year-old woman (patient 2) who died 2 days before the child after a brief illness characterized by progressive shortness of breath, cough, abdominal pain, fever, and hemoptysis. Histopathologic findings for the lungs of patient 2 resembled those identified for patient 1 (Figure, panels C, D), but there was no evidence of SARS-CoV-2 in the lung tissues by RT-PCR. Because clinicopathologic features were characteristic of HPS, we performed IHC assay for hantavirus. IHC showed typical punctate granular staining of hantaviral antigens in pulmonary and glomerular capillaries, characteristic of HPS (4) (Figure, panels E, F). IHC evaluation of lung and kidney tissues of patient 1 for hantavirus showed a similar pattern, confirming the infection in both patients (Figure, panel A, right side).

The clinicopathologic and IHC findings indicate that both patients died from HPS. Although SARSCoV-2 RNA was detected by RT-PCR in patient 1 , it was not the probable underlying cause of death. This scenario provides an essential reminder that previously recognized, nonendemic infectious diseases that clinically resemble COVID-19 continue to occur during the pandemic, in a manner similar to other clinicopathologic mimics described previously during other pandemic diseases (7).

Consideration of alternative diagnoses of diseases that precipitate acute respiratory distress syndrome and co-infections remains crucial for diagnosing and treating of critically ill patients, as well as accurately determining causes of death. For HPS, triage tools such as peripheral blood smear review and identifying 4 of 5 findings (thrombocytopenia, hemoconcentration, granulocytic left shift, absence of toxic changes, and $>10 \%$ immunoblasts) can be used 


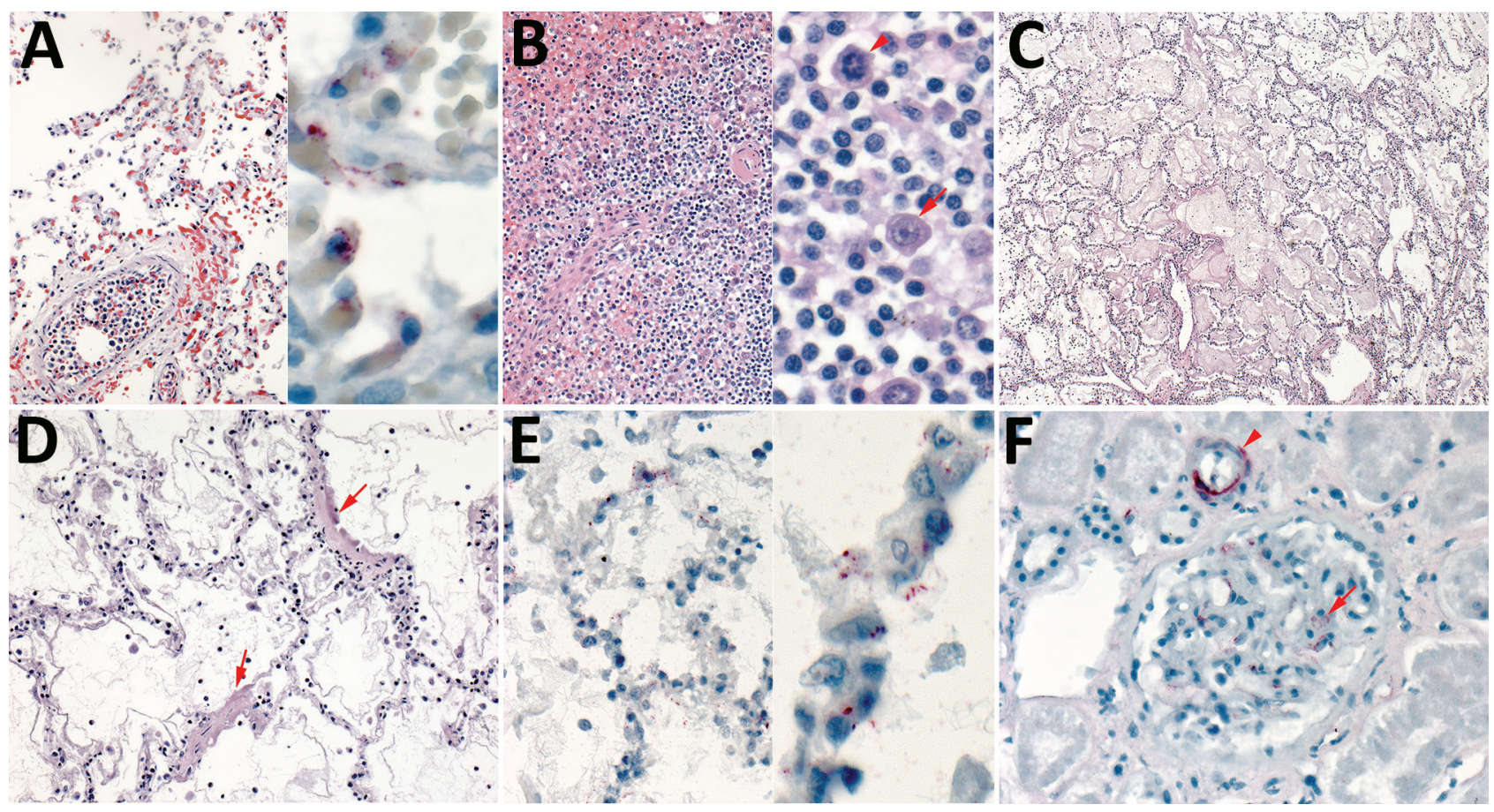

Figure. Histopathologic and immunohistochemical characteristics of fatal hantavirus pulmonary syndrome in 2 patients, Arizona, USA, 2020. A) Patient 1 lung tissue, showing intravascular leukocytosis with left shift (left, original magnification $\times 50$ ) and hantavirus antigen immunostaining (red) in pulmonary microvasculature (right, original magnification $\times 158$ ). B) Patient 1 spleen tissue, showing immunoblast proliferation in the red pulp and periarteriolar sheaths (left, original magnification $\times 50$ ) and immunoblasts with high nuclear to cytoplasmic ratio, vesicular and prominent nucleoli (arrows) and mitosis (arrowhead) (right, original magnification $\times 158$ ). C) Patient 2 lung tissue, showing severe intraalveolar edema (original magnification $\times 12.5$ ). D) Patient 2 lung tissue, showing interstitial pneumonitis with hyaline membranes (arrows) (original magnification $\times 50$ ). E) Patient 2 lung tissue, showing hantavirus antigen immunostaining (red) in pulmonary microvasculature (left, original magnification $\times 50$; right, original magnification $\times 158$ ). $F$ ) Patient 2 kidney tissue, showing hantavirus antigen immunostaining (red) in glomerular capillaries (arrowhead) and interstitial vessel (arrow) (original magnification $\times 100$ ).

to diagnose the disease rapidly and presumptively in the clinical setting $(8,9)$. Communication and partnerships of local, state, and federal public health officials and healthcare professionals, including clinicians, infectious disease specialists, pathologists, and medical examiners, are essential during these challenging times of the SARS-CoV-2 pandemic.

This study was supported by the Coordenação de Aperfeiçoamento de Pessoal de Nível Superior Brazil (Finance Code 001; doctoral scholarship for T.M.W.).

\section{About the Author}

Ms. Wilson is a guest researcher, as part of a doctoral scholarship from the Capes-PrInt Program, in the Division of High-Consequence Pathogens and Pathology, National Center for Emerging and Zoonotic Infectious Diseases, Centers for Disease Control and Prevention, Atlanta, GA. Her research interests include investigative and comparative pathology and pathogenesis of zoonotic and human infectious diseases.

\section{References}

1. Zhu N, Zhang D, Wang W, Li X, Yang B, Song J, et al.; China Novel Coronavirus Investigating and Research Team. A novel coronavirus from patients with pneumonia in China, 2019. N Engl J Med. 2020;382:727-33. https:/ / doi.org/ 10.1056/NEJMoa2001017

2. Duchin JS, Koster FT, Peters CJ, Simpson GL, Tempest B, Zaki SR, et al.; The Hantavirus Study Group. Hantavirus pulmonary syndrome: a clinical description of 17 patients with a newly recognized disease. N Engl J Med. 1994;330:949-55. https://doi.org/10.1056/ NEJM199404073301401

3. Nichol ST, Spiropoulou CF, Morzunov S, Rollin PE, Ksiazek TG, Feldmann H, et al. Genetic identification of a hantavirus associated with an outbreak of acute respiratory illness. Science. 1993;262:914-7. https:/ / doi.org/10.1126/ science. 8235615

4. Zaki SR, Greer PW, Coffield LM, Goldsmith CS, Nolte KB, Foucar K, et al. Hantavirus pulmonary syndrome: pathogenesis of an emerging infectious disease. Am J Pathol. 1995;146:552-79.

5. Martines RB, Ritter JM, Matkovic E, Gary J, Bollweg BC, Bullock H, et al.; COVID-19 Pathology Working Group. Pathology and pathogenesis of SARS-CoV-2 associated with fatal coronavirus disease, United States. Emerg Infect Dis. 2020;26:2005-15. https://doi.org/10.3201/ eid2609.202095 
6. Centers for Disease Control and Prevention. Collection and submission of postmortem specimens from deceased persons with known or suspected COVID-19, November 2020 (Interim Guidance) [cited 2020 Oct 21]. https:/ / www.cdc.gov/ coronavirus/2019-ncov/hcp/guidance-postmortemspecimens.html

7. Blau DM, Denison AM, Bhatnagar J, DeLeon-Carnes M, Drew C, Paddock C, et al.; Infectious Diseases Pathology Branch Working Group. Fatal infectious diseases during pandemic (H1N1) 2009 outbreak. Emerg Infect Dis. 2011;17:2069-70. https://doi.org/10.3201/eid1711.110429

8. Koster F, Foucar K, Hjelle B, Scott A, Chong YY, Larson R, et al. Rapid presumptive diagnosis of hantavirus cardiopulmonary syndrome by peripheral blood smear review. Am J Clin Pathol. 2001;116:665-72. https:/ / doi.org/ 10.1309/CNWF-DC72-QYMR-M8DA

9. Dvorscak L, Czuchlewski DR. Successful triage of suspected hantavirus cardiopulmonary syndrome by peripheral blood smear review: a decade of experience in an endemic region. Am J Clin Pathol. 2014;142:196-201. https:/ /doi.org/ 10.1309/AJCPNFVWG46NUHED

Address for correspondence: Sherif R. Zaki, Centers for Disease Control and Prevention, 1600 Clifton Rd NE, Mailstop H18-SB, Atlanta, GA 30329-4027, USA; e-mail: szaki@cdc.gov

\section{Novel Mutation of SARS-CoV-2, Vietnam, July 2020}

\author{
Hoang Vu Mai Phuong,,$^{1}$ Trinh Son Tung, ${ }^{1}$
} Ung Thi Hong Trang, Nguyen Le Khanh Hang, Nguyen Vu Son, Pham thi Hien, Le thi Thanh, Vuong Duc Cuong, Ton That Thanh, Nguyen thi Thanh Nhan, Tran Nhu Duong, Ngu Duy Nghia, Tran Anh Tu, Marc Choisy, Maia A. Rabaa, H. Rogier van Doorn, Dang Duc Anh, Le Quynh Mai

\begin{abstract}
Author affiliations: National Institute of Hygiene and Epidemiology, Hanoi, Vietnam (H.V.M. Phuong, U.T.H. Trang, N.L.K. Hang, N.V. Son, P. thi Hien, L. thi Thanh, V.D. Cuong, T.N. Duong, N.D. Nghia, T.A. Tu, D.D. Anh, L.Q. Mai); Oxford University Clinical Research Unit, Hanoi (T.S. Tung, H.R. van Doorn); Centre for Disease Control and Prevention, Danang, Vietnam (T.T. Thanh, N. thi T. Nhan); Oxford University Clinical Research Unit, Ho Chi Minh City, Vietnam (M. Choisy, M.A. Rabaa); University of Oxford, Oxford, UK (M. Choisy, M.A. Rabaa, H.R. van Doorn)
\end{abstract}

DOI: https://doi.org/10.3201/eid2705.210013

${ }^{1}$ These authors equally contributed to this work.
A cluster of severe acute respiratory syndrome coronavirus 2 infections in Danang, Vietnam, began July 25,2020 , and resulted in 551 confirmed cases and 35 deaths as of February 2021. We analyzed 26 sequences from this cluster and identified a novel shared mutation in nonstructural protein 9, suggesting a single introduction into Vietnam.

$\mathrm{V}$ ietnam experienced 2 clusters of severe acute respiratory syndrome coronavirus 2 (SARSCoV-2) infections during January 23-April 15, 2020 (270 cases, 163/270 imported) (1-4). After 99 days without community transmission, a cluster of SARSCoV-2 infections of unknown origin was detected in Danang; it was found in Danang General Hospital on July 25 in a 57-year-old male patient (DN001) experiencing pneumonia who had no travel history. During a subsequent round of contact tracing, 14 additional SARS-CoV-2-positive cases were detected both in the community $(\mathrm{n}=3)$ and Danang hospitals $(\mathrm{n}=11)$. Vietnam then initiated large-scale contact tracing and quarantining. A total of 551 confirmed cases were reported from 15 cities and provinces across the country; 540 (98\%) either were related to major hospitals in Danang or were in patients who had visited Danang during July 25-September 3. This cluster included 35 COVID-19 fatalities, most (32) hospital-acquired and associated with concurrent conditions or old age $(2,5)$. Danang General Hospital, the epicenter of the outbreak, reported 246 cases among inpatients, caregivers, and healthcare workers. Strict prevention measures of contact tracing, quarantine, and isolation were again implemented nationally, and the outbreak was successfully contained. We describe the molecular epidemiology of this cluster.

We performed sequencing of 26 nasopharyngeal or throat swab specimens that were sent to the National Institute of Hygiene and Epidemiology (Hanoi, Vietnam) for diagnostics; all were positive for SARS-CoV-2 and had cycle threshold value $<30$ by real-time reverse transcription PCR (6-8). Of those specimens, 18 were collected from patients in hospitals and communities in Danang and the rest from outside Danang: Ha Nam (1), Quang Nam (1), Thanh Hoa (1), Hanoi (2), Lang Son (2), and Hai Duong (1). We uploaded sequences to the GISAID database (https://www.gisaid.org; accession nos. 759869-91 and 766029-31).

All 26 sequences belonged to lineage B.1.1 and clustered together in the global tree, with the exception of DN013 (Figure 1, panel A). DN013 contains an additional single-nucleotide polymorphism 